\title{
Comparison of Test Methods to Determine Failure Parameters for MAT162 Calibration
}

\author{
Matthew Molitor ${ }^{1}$, Brian Justusson ${ }^{1}$ \\ Boeing Research and Technology, Berkeley, MO 63134 \\ Mostafa Rassaian², Jenna Pang ${ }^{3}$ \\ Boeing Research and Technology, Tukwila, WA 98108
}

Subject Category: Structures, Progressive Failure Analysis, Impact

\begin{abstract}
MAT162, a laminated composite failure material model developed by The Material Sciences Corporation for the commercial finite element software LS-Dyna, is widely used within the aerospace industry to predict damage events under a range of dynamic conditions. The material model involves numerous inputs consisting of both physical material properties and numerical calibration parameters. Due to the large number material card inputs, often there is a lack of uniqueness to MAT162 material cards that limits the predictive capability to only the directly calibrated space. To expand this space, MAT162 requires a prudent and robust calibration process in which significant parameters are calibrated to high confidence damage events observed in experiment. Critical to this success is fully defining the material properties correctly, namely the fiber crush (SFC) and fiber shear (SFS) values, prior to calibrating the numerical parameters. In this paper, the effect of the determination of SFS and SFC on subsequent calibration steps is examined using two different experimental techniques.
\end{abstract}

\section{Introduction}

Progressive Damage and Failure Analysis (PDFA) methods have been developed for a number of loading applications including static, fatigue, and impact. The technical maturity of these methods is related to the capability of the codes to predict damage for the loading case and is tied to the understanding of the relevant physics for that case. PDFA methods have been shown to be technically mature for a number of static applications including open-hole tension and compression [1] [2] and low velocity impact [3] [4]. Fatigue capable PDFA tools currently require additional analysis and development as intralaminar fatigue becomes better understood. High energy dynamic impact (HEDI) applications also require additional material modeling method development due to a wide variety of material and structural level phenomena that either do not exist at static loading rates or become much more significant due to strain rate effects.

The NASA Advanced Composites Consortium (ACC) seeks to develop PDFA tools to reduce the timeline for certification of new composite aircraft structures. Further, the HEDI Team seeks to develop novel PDFA methods and to create industry standards for best practices for evaluating and using these methods for a variety of high energy impact events. An overview of the NASA ACC HEDI effort including the use of LS-DYNA MAT162, LS-DYNA MAT261, Smoothed Particle Galerkin (SPG), and EMU Peridynamics to HEDI was presented [5] with detailed

\footnotetext{
${ }^{1}$ Structural Engineer, Boeing Research \& Technology, 6300 James S. McDonnell Blvd. Berkeley, MO 63134, NonMember.

${ }^{2}$ Boeing Technical Fellow, Boeing Research \& Technology, 9725 E Marginal Way S, Tukwila, WA 98108-4040, AIAA Associate Fellow

${ }^{3}$ Structural Engineer, Boeing Research \& Technology, 9725 E Marginal Way S, Tukwila, WA 98108-4040, NonMember
} 
methods and results for LS-DYNA MAT261 [6] and EMU Peridynamics [7]. The focus of this paper is the comparison of material test methods used to obtain failure properties unique to LS-DYNA MAT162.

LS-DYNA MAT162 [8] [9] is considered an industry standard for prediction of damage during HEDI events with composite material. The model is attractive for this application because it includes material input properties critical to impact performance that many less mature material models do not have. These material input properties include strain rate effects, failure modes only observed at elevated loading rates and a native representation of delamination behavior. MAT162 contains two failure modes that are generally not observed under low loading rates (e.g. static or low energy impact such as BVID). These failure modes are the fiber shear strength (SFS) and the fiber crush strength (SFC).

MAT162 contains a number of physical material properties to represent elastic behavior and to initiate failure, but requires a robust calibration scheme. Several ways to calibrate the post-peak response (often referred to as the softening curves) are proposed including the Quasi-Static Punch Shear Test (QS-PST) [10] and Low Velocity Impact (LVI) [11] in which predictable damage morphologies develop and progress. In order to achieve predictive capability within the desired design space, it is necessary to properly determine the material failure (damage initiation values) properties prior to calibration, however, there are difficulties in determining the SFS and SFC properties.

In this paper, two experimental procedures are examined to determine the failure parameters SFS and SFC for an IM7/8552 tape material system. The SFS and SFC values obtained from these two experiments will be used to populate a MAT162 card, which will subsequently be calibrated to a QS-PST. A comparison will be made between the calibration results, specifically in regard to the effects of the SFS and SFC values used. Finally, best practices and lessons learned will be presented.

\section{LS-DYNA MAT162 Material Model Description within PDFA Framework}

PDFA methods attempt to develop physics-based constitutive models to accurately describe the response of a composite material at the length scale of interest (e.g. fiber/matrix, lamina, or laminate) [12]. For MAT162, this is performed at the lamina level for either a fabric or a tape material system. The general lamina level offers a relevant length scale for the mechanics and is validated with experimental data. Figure 1 shows the four responses that are included in constitutive models proposed by Razi et al [12]. For the lamina level response, the domain can be discretized into a representative volume element. Of note, the material model presented in Figure 1 gives a comprehensive overview of the material model implementation that is not specific to a method, but rather describes the response in a method,scale or class independent way.

A general PDFA response includes four main regions:

A. Elastic Response: The elastic properties are one of the major inputs for PDFA methods. In general, it is best to characterize all nine elastic properties to provide the highest confidence in the constitutive model, however, assumptions exist to limit the necessary testing (e.g. transverse isotropy for a traditional tape system).

B. Pre-Peak Response: The pre-peak response determines how the model represents nonlinearity that occurs as a result of damage at a length scale below the scale of interest. In general, this nonlinearity has been shown to have both elastic/recoverable nonlinearities as well as non-recoverable damage. The sophistication of the constitutive model of the pre-peak response ultimately governs how much of this energy is dissipated or recoverable.

C. Failure Criteria: PDFA methods have a number of failure modes that are characterized by failure stresses. In order to know that an element has failed, a relevant failure criteria is necessary for each mode of failure. Many methods have traditional matrix interaction failure criteria, however, in high velocity impact, modemixity laws do not exist or are not fully matured.

D. Post-Peak: The post-peak response governs how energy is dissipated from a a representative unit cell after the failure criteria is met. The post-peak response can have one of three forms:

1. Instantaneous energy degradation where energy is instantly removed from the system by element deletion or similar method. Another form of instantaneous energy degradation is through the use of a finite slope of an unloading curve that is not linked to a physical material property.

2. Energy-based degradation where fracture-like processes take place and dissipate the energy through an apparent fracture. This type of degradation uses traction-separation laws in which the area under the curve is equal to the fracture energy in the failure mode of interest. 
3. Calibrated energy dissipation where a post-peak softening curve is calibrated to a specific test to represent a range of behaviors such as fiber failure, matrix damage and delamination.

MAT162 is considered a PFA method as it does not include the pre-peak damage accumulation and only contains regions A, C, and D. As a fully 3D method, MAT162 requires all nine elastic constants (region A). MAT162 includes all failure criteria (region C) for the orthogonal tension and compression, the three shear failure modes, and SFS and SFC. Finally, MAT162 has parameters AM1-AM4 that are used for calibration. AM1-AM4 are post-peak numerical softening parameters that are used to calibrate to a material response (region D). These parameters are calibrated to represent energy dissipation as a function of the elastic pre-peak energy for a given volume, and are not directly energy-based nor instantaneous energy degradation algorithms.

Unique to MAT162 are the SFS and SFC failure modes that are generally considered a higher order failure modes that are a result of localization and/or loading rate effects. SFS represents the average transverse shear stress through the thickness of a fiber at the point the fiber fails in pure shear. The MAT162 fiber failure criteria uses a quadratic interaction between fiber shear and fiber tension to determine the onset of damage. For a fiber oriented in the 'aa' direction, SFS is compared to the fiber shear stress obtained by the following equation:

$$
\tau_{\text {Fiber_Shear }}=\sqrt{G_{a b}^{2} \varepsilon_{a b}^{2}+G_{c a}^{2} \varepsilon_{c a}^{2}}
$$

where $\mathrm{G}$ is shear stiffness and $\varepsilon$ is shear strain.

SFC represents the through the thickness compression stress at which the fiber fails. For a fiber oriented such that the 'cc' direction is through the thickness, SFC is compared to the fiber crush stress obtained by the following equation:

$$
\sigma_{\text {Fiber_Crush }}=E_{C}\left\langle-\varepsilon_{C}\right\rangle
$$

where $\mathrm{E}$ is through-thickness axial stiffness and $\varepsilon$ is through-thickness axial strain.

Delaminations can be handled in one of two ways. The first way involves using the native delamination feature of MAT162. The MAT162 method requires a mesh which uses three elements through the thickness of each part (lamina) coupled with an element softening scheme to replicate nodal release, however, it is not an energy-based release. Conversely, native tie-break contact can be used between layers that offers a fracture-based release and is a commonly used technique within industry to have one-element-per-ply representation of the damage. For this work, tie-break contact is used; however, it is worth noting that the MAT162 failure criteria does not allow for the ability to turn off the native delamination capabilities. At the completion of the analysis cases, checks were performed to determine if the native delamination had been triggered during the calibration.

Of note, MAT162 is a PFA method that attempts to capture total energy dissipation through an impact event, however, it can be calibrated further to a length scale of interest. There exist coupling coefficients between failure modes for both the fabric and tape material forms; however, the post-peak softening parameters can easily be calibrated to a length scale of interest. It is not uncommon for a MAT162 part to represent a sub-laminate made up of multiple offaxis plies. In addition to losing the coupling of failure modes of interest through the damage mode correlation based on material form, the damage becomes more smeared through the thickness of the sub-laminate and it is therefore more difficult to discern indivual failure modes.

\section{Test Methods to Determine Fiber Shear Mode Strength (SFS) and Crush Strength (SFC)}

Currently, there are two test procedures that have been proposed to determine SFS and SFC directly without the need for model calibration. The original standard called for a modified QS-PST in which the span-to-punch ratio is set to a predetermined ratio to obtain data for either SFS or SFC. Due to difficulties analyzing the data to determine the material property, a second test was developed known as the Laterally Constrained Compression Test (LCCT). The following subsections outline the test procedures and considerations for each of the methods for determining SFS and SFC.

\section{III.a Quasi-Static Punch Shear (QS-PS) Test}

American Institute of Aeronautics and Astronautics 
Figure 2 shows a schematic of a typical QS-PST fixture. Of note, there are two critical dimensions: the Punch Diameter, $\mathrm{D}_{\mathrm{p}}$, and Support Span, $\mathrm{D}_{\mathrm{s}}$. The ratio of the support span to the punch diameter is called the span-to-punch ratio (SPR). To determine the fiber crush strength, the SPR is set to 0 . This is done by putting a flat plate behind the sample and punching with a standard punch. To determine the fiber shear strength, the SPR was set to 1.1. A test matrix is shown in Figure 3. Best practices for testing are listed as follows:

1. The punch edges should be flat and not rounded with the punch made out of a hardened steel

2. The sample shall be loaded at a rate of $0.05 \mathrm{in} . / \mathrm{min}$ via actuation of the punch.

3. It is recommended that the test engineer shall perform a minimum of five (5) tests for each of the SPR ratios to obtain the material behavior of interest.

4. For SFS, if delamination is observed, the thickness of the punch lip should be determined to properly determine the shear stress.

5. For SFC, the load-deflection response should be examined for deviations from linearity that is characteristic of a fiber crush.

\section{III.b Laterally Constrained Compression Test (LCCT) Test}

A block of material is loaded in compression through the thickness while the transverse direction is constrained as described by Yen and Pankow [13] [14]. A shear band forms through the specimen during failure. The angle of the shear band in conjunction with the ultimate load is used to determine the SFS and SFC strength values. See Figure 4 for a visual of the test setup.

Best practices for testing are listed as follows:

1. The sample must be ground flat and parallel to within 0.001" on the sides that are being constrained and loaded.

2. The loads are expected to be high, as such, the fixture should be designed to handle the loads. The fixture must be made from a hardened steel.

3. Sample should be loaded at a rate $0.5 \mathrm{in} / \mathrm{min}$ ensuring that the loading punch is square and not contacting the sides of the test fixture.

4. Careful measurements should be taken after failure to determine the fracture angle.

\section{Experimental Results}

Experiments to determine SFS and SFC were performed on the IM7/8552 material system for the QS-PST and LCCT test configuration. The QS-PST testing was performed at the Boeing Research \& Technology Fast Lean Test Cell located in Tukwila, Washington. The LCCT testing was performed at North Carolina State University's Ballistic Loading and Structural Testing (BLAST) Lab. A comparison of the results is shown in Table 1 which shows that the LCCT testing produced values for SFS and SFC that were 30-40\% higher than what was determined by the QS-PS Test. The following sections outline the experimental results and determination of the SFS and SFC parameters.

\section{IV.a Quasi-Static Punch Shear (QS-PS) Test Results}

The first series of tests performed were for the $S P R=0.0$ tests with the fixture backed with a flat plate to create a fully supported span underneath the sample. The load deflection curves for the SPR $=0.0$ are shown in Figure 5. Testing did not reveal a clear load drop, however, this behavior is expected as a hydrostatic state of stress is developed underneath the loading head. During the loading, there were characteristic small load drops that are consistent with an individual ply failing through a crush behavior. After each of the load drops, the punch displacement continued to load the panel. The final damage state of a representative IM7/8552 test panel is shown in Figure 6.

The first ply failure load drop was used in conjunction with the punch contact area to determine the fiber crush stress, SFC. The SFC failure stress is calculated from the following equation:

$$
S F C=F_{1 s t} / A_{\text {PUNCH }}
$$

Where $F_{1 s t}$ is the load at which the first drop in the curve occurs and Apunch is the cross sectional area of the punch. The fiber crush stress determined from QS-PST results for the IM7/8552 tape material system was SFC = 160.6 +/3.7 ksi. Tabulated results of SFC calculations are shown in Table 2. 
The next series of tests performed was for the SPR=1.1 scenario. This SPR ratio is used to determine the strength of the ply by shearing through the thickness. For a tape system, this allows for shearing through the unidirectional ply. The QS-PST fixture was backed with a flat plate with a circular opening of 2.0 in concentric with the center line of the punch. The punch had a diameter of 1.8 in. The load-displacement curves for the SPR=1.1 scenario (Figure 7) show the characteristic response of the material.

Within these curves, there are two points of interest. The first is a characteristic load drop that occurs at a displacement of $0.02 \mathrm{in}$. This load drop is the result of an internal delamination. After that delamination forms, the plies begin to shear further until the final large load drop is observed. The load drop is consistent with a shear plug being developed and pushed through the back surface. Figure 8 shows a representative sample after failure. From this, it is clear that delamination occurs internally as there is a lip of material present on the shear plug. After failure, the plug pushes all the way through the sample.

At the completion of the test, the thickness of the lip was measured three (3) times using a micrometer. This allowed for determination of the depth of the delamination of as a result of the punch shear testing. These measurements were averaged and the result is shown in Table 3. This thickness represents the portion of the laminate that fails in membrane tension after the shear plug is formed. So the thickness that fails in fiber shear would be equal to the remaining portion of the laminate thickness.

The peak load prior to the final large load drop is used in conjunction with the thickness measurements of the lip on the shear plug to calculate a shear failure stress, SFS. It is assumed that the large load drop is due to the formation of a shear plug on the punch side of the laminate. The small load increase and subsequent curve runout is the material remaining after the formation of the shear plug undergoing combined membrane tension and punch shear. The SFS failure stress is calculated from the following equation:

$$
S F S=F_{\text {PEAK }} / A_{\text {PLUG }}
$$

Where $F_{\text {PEAK }}$ is the peak load from the curve shown in Figure 7 and $A_{P L U G}$ is the calculated based on the circumference of the punch and the thickness of the shear plug determined from measured coupon thickness and the resulting lip thickness in Table 3. The fiber shear stress determined from QSPS test results for the IM7/8552 tape material system was SFS $=38.5+/-3.0 \mathrm{ksi}$. Tabulated results of SFS calculations are shown in Table 4 .

\section{IV.b Laterally Constrained Compression Test (LCCT) Test}

The determination of the material properties are described in Pankow et al. [13]. At failure, a shear plane forms through the material (Figure 9). The plane occurs at a prescribed angle $(\theta)$ that is a result of the material properties. Of interest are the state of compression stress (SFC) and the normal and shear components along the shear plane. Figure 10 shows the typical failure response of a tape material, notably the development of a clear shear plane. The crush strength (SFS) is determined from the following equation:

$$
S_{F C}=F_{\max } / A
$$

Where $\mathrm{F}$ is the maximum force recorded by the load frame and $\mathrm{A}$ is the cross sectional area. The normal and shear forces can be determined from the following set of equations:

$$
\begin{array}{r}
S_{N}=S_{F C} \cos ^{2} \theta \\
S_{N F S}=\frac{1}{2} S_{F C} \sin 2 \theta
\end{array}
$$

Using a Mohr's circle transformation with the assumption that SFC and 0 are the two principle stresses, SFS can be determined from the following equations: 


$$
\begin{gathered}
S_{F S}=S_{N F S}+\mu S_{N} \\
\mu=\tan \phi \\
\phi=90^{\circ}-2 \theta
\end{gathered}
$$

SFS and SFC were determined to be $52.7 \pm 10.5 \mathrm{ksi}$ and $207.6 \pm 19.9$ ksi respectively.

\section{Preliminary Modeling Results}

The calibration of LS-DYNA MAT162 numerical softening parameters begins with correlation of test data to model results of the QS-PST. Typical MAT162 calibration relies on performing QS-PST with both a characteristically thick and a characteristically thin panel. Characteristic thickness, $\mathrm{T}$, is defined as:

$$
\mathrm{T}=\frac{\mathrm{D}_{\mathrm{P}} \mathrm{D}_{\mathrm{S}}}{\mathrm{T}_{\mathrm{L}}^{2}} \quad \mathrm{~T}>100 \Rightarrow \text { Thin Laminate } \quad \mathrm{T}<100 \Rightarrow \text { Thick Laminate }
$$

A characteristically thick specimen is selected to test because it prodcuces repeatable and predictable sequential failure modes that are representative of those seen in a ballistic impact. Characteristically thin laminates may be tested as well, but tend to experience multiple modes of damage simultaneously and undergo very rapid drops in load which makes calibration of individual softening parameters difficult. Characteristically thin laminate tests do serve well as a low-level validation of the calibration effort that is performed on the characteristically thick laminate. The IM7/8552 tape material system exhibited consistent behavior in the QS-PST with an SPR $=4.0$ on a characteristically thick laminate, and so the force-displacement results of the (5) test runs were averaged. This was done in order to calibrate to an average response as opposed to doing a calibration to a single data point. Test fixture compliance was measured and accounted for in the development of the average response. Figure 10 shows the raw force-displacement data and the curves representing the average value and the compliance-adjusted average value to be used for correlation.

The QS-PST data of thick laminates is particularly useful for calibrating MAT162 softening parameters AM1-AM4 due to the sequential nature of the initiation and progression of damage modes. Figure 11 shows the average test data used for calibration, and identifies the damage modes and softening parameters associated with each portion of the curve. When performing calibration it is expected that all stress limits will be known values, which limits the variability of the model response to the post-peak calibration of the softening parameters. Due to the sequential nature of damage initiation, progression, and load redistribution it is critical that the model appropriately represent the behavior of the beginning portion of the load-displacement curve before attempting to move to subsequent portions. This ensures that the state of stress and location of damage allows the next mode of damage to occur in the correct location at the correct point in the punch displacement.

The first step of the QS-PST calibration is to adjust the strain softening parameter AM4 to account for the behavior of through-thickness and in-plane matrix damage. The AM4 softening parameter controls the post-peak behavior of elements that have damaged due to in-plane shear, through-thickness shear, and delamination. The associated portion of the punch force-displacement curve affected by AM4 is shown in Figure 11. The SFS and SFC limit values are expected to be known at this point in the calibration process as they define the initiation point of the second major damage modes. The softening behavior calibrated with AM4 affects the load-carrying capability of elements damaged in shear, and so plays a large role in the load distribution at the initiation point of the next damage mode. Therefore, AM4 has a direct impact on the point at which the SFS and SFC limit values are reached.

Figure 12 shows the calibration attempts for the softening parameter AM4 using preliminary estimates for fiber shear stress (SFS=30ksi) and fiber crush stress (350ksi) prior to the availability of test data. Fiber shear failure occurs prior to the first peak in test data. This indicates that the estimated value of SFS=30ksi is too low, as it allows fiber failure to occur prior to the completion of the first matrix-only damage mode in the test. Decreasing values of AM4 enable greater post-peak shear capability after the initiation of matrix damage, which tends to mask the behavior of the low estimated SFS value. 
Figure 13 shows the calibration attempts for AM4 using SFS and SFC values obtained from LCC testing. The variation in the model result curves shows the sensitivity of the model to the post-peak behavior of one failure mode. The response of all model runs using various AM4 values in Figure 13 indicates that the values of SFS and SFC are sufficiently high to allow matrix damage and delamination to occur prior to fiber damage. The curve using AM4=4 is promising, as it captures the nonlinear behavior, softens during delamination, then recovers loading up to approximately the same value as the peak seen in testing. For these values of SFS and SFC, further calibration of AM4 values greater than 4 is warranted to evaluate greater amounts of softening due to matrix damage. The next step would be to determine the influence of the AM3 softening parameter, which controls the post-peak behavior of the next sequential damage mode, fiber crush.

Figure 14 shows the calibration attempts for AM4 using SFS and SFC values obtained from QS-PS testing. The response of the model runs using various AM4 values in Figure 14 indicates that the value of SFS was slightly too low. Fiber shear damage occurs concurrently with matrix damage and initial delamination failure. The value of SFC is sufficiently high to allow for the accumulation of matrix damage prior to fiber crush damage at the second peak in the curve. The curve using AM4=2 is promising, as it captures the initial nonlinear behavior, softens during delamination, then recovers load up to approximately the same value as the peak seen in testing. However, AM4=2 does not allow for softening to the extent that test data indicates after the first peak. The curve using AM4 $=4$ shows more softening after the first peak, however, it does not quite reach the first peak. The AM4=4 curve also experiences an abrupt load drop prior to reaching the displacement at peak load seen in the test data. For these values of SFS and SFC, values of AM4 between 2 and 4 appear to sufficiently represent the initial behavior of the average QSPS test data. Further calibration is warranted by investigating the influence of the AM3 softening parameter.

The influence of failure stress limits SFS and SFC on model behavior can be even larger than the value of the softening parameter AM4. In the QS-PS test, the fiber shear and/or crush mode of damage follows matrix damage sequentially as the second damage initiation mode. Altering SFS and SFC allows for a large difference in stress distribution at the point of fiber shear or fiber crush damage initiation. A comparison of calibration curves using the various values of SFS and SFC with an AM4 value of 4.0 is shown in Figure 15. Higher values enable further accumulation of matrix damage prior to fiber damage. Lower values tend to limit the extent of matrix damage prior to fiber damage, as the crush or shear mode initiates earlier. This truncates the peak load with an abrupt load drop.

The QSPS model is also used to calibrate softening parameters AM1-3, and the element erosion parameter E_LIMT in a sequential process. The results of a QS-PST calibration including all softening parameters, AM1-AM4, can be seen in Figure 16. Element erosion parameters have not yet been calibrated, and so the model run was truncated prior to load runout. The model response is in good agreement with the average test data curve. Both the qualitative and quantitative behavior of the model match the test data well.

\section{Discussion}

LS-DYNA MAT162 has been shown to perform well for predicting impact damage on composite laminates in high energy events only if it is calibrated to the length scale of interest. Calibration of multiple softening parameters can be performed using the sequential progression of damage modes typical in QS-PST of characteristically thick composites. All stress limits must be well-defined prior to calibration to ensure that the initiation of each damage mode occurs at the appropriate state of stress. SFS and SFC are stress limits that are unique to MAT162, and typically present themselves as significant modes of failure only in high energy impact events

Obtaining values for SFS and SFC is difficult using quasi-static tests as other more prevalent material damage behavior must be prevented to isolate the damage mode of interest. Two test methods were proposed to obtain SFS and SFC values - QS-PST and LCC.

The data obtained from QS-PST (SPR=1.1) was used to calculate SFS. Of note, the first failure in the coupons was not the failure mode of interest. Each coupon experienced delamination prior to fiber shear failure. This required measuring the thickness of the resultant lip on the failed shear plug to calculate the area of fiber shear failure. The scatter in calculated SFS values was low, SFS $=38.5+/-3.0 \mathrm{ksi}$. However, using this value in QS-PST calibration models revealed that fiber shear failure occurred prior to major matrix damage and delamination modes of failure, which indicates that the calculated value is likely too low. To improve future test results using this method, it is

American Institute of Aeronautics and Astronautics 
desirable to prevent the delamination mode of failure, and to create a stress state in the fibers as close to pure shear as possible. To that end, it is recommended to use a thinner laminate and approach an SPR value closer to 1.0.

QS-PST (SPR=0.0) results do not present a clear point of fiber crush failure, and so the load value at which failure occurs must be selected based on judgment. The load-displacement curves exhibit multiple small load drops of varying magnitudes. These load drops are difficult to associate with a known amount of fiber crush failure, as it is unclear the extent to which the specimen has failed. It is possible that multiple lamina have failed in a small area near the edge of the punch, or that a single lamina has crushed uniformly across the entire area of the punch. Some fiber shear failure is likely occurring at the edge of the punch as well. The scatter in calculated SFC values was low, SFC $=160.6+/-3.7 \mathrm{ksi}$. Using this value in QS-PST calibration models revealed that it is reasonable, though likely too low. It is recommended that thinner laminates and a hardened steel punch and backing plate be used to determine the value of SFC.

The SFS and SFS values obtained from LCC testing provided the most encouraging model response during calibration of the AM4 strain softening parameter for MAT162. Processing the LCC test results requires measurement of the failure angle formed by the fracture plane through the specimen. This measurement can be difficult to obtain due to the tendency of the material to crush into powder. The scatter in resulting SFS and SFC values was fairly large, as SFS and SFC were determined to be $52.7 \pm 10.5 \mathrm{ksi}$ and $207.6 \pm 19.9 \mathrm{ksi}$ respectively. This scatter can be equally attributed to variations in measured fracture angle and maximum load at failure. LCC testing requires the use of hardened steel press and fixturing, as the compression stresses required to fail the specimen are large enough to yield softer materials. It is important to ensure that coupons are machined with flat and parallel edges so that uniform stress states are generated. Where applicable, it is recommended to include digital image correlation on the exposed face of the specimen so that the failure angle may be captured prior to total crushing failure.

Preliminary modeling results showed the influence of SFS and SFC values on the calibration of AM4, generally considered the first calibration parameter for the QS-PST. SFS and SFC should not play a role in the early portion of the curve where AM4 is being calibrated, as the fiber shear and fiber crush damage modes have not occurred at this point in the test. The curves generated using estimated values of SFS and SFC exemplify this behavior. This leads to an improperly calibrated AM4 parameter to overcome the poorly defined SFS and SFC stress limits. The resulting state of damage and distribution of stress is not representative of physical material behavior, and so the predictive capabilities of the progressive failure model fall short as the problem begins to cascade. AM4 calibration model curves generated using SFS and SFC values generated via LCC match well with average QS-PST ( $S P R=4.0$ ) test data up to the point where AM3 calibration would begin. For future efforts, it is recommended to use LCC testing to acquire SFS and SFC stress limits.

\section{Remarks and Future Work}

This paper highlights the difference between two test methods to determine failure strengths that are typically observed only under high strain rates of loading and/or localization. These failure modes are of particular importance because they become critical for proper prediction of ballistic impact performance. This paper shows how the selection of these parameters affects the downstream calibration effort for a fully populated MAT162 card.

Current work being performed under NASA ACC Phase II is utilizing calibrated MAT162 cards to execute pre-test model predictions for a variety of high rate structural and impact loading events. These predictions serve to inform detailed testing configurations, ensuring the desired mode of failure and collection of meaningful data are achieved. Parametric studies are being performed to determine the effects of multiple test variables in an effort to reduce the overall test matrix.

The length scale discussed in the paper is the material model length scale, not the size of the components being modeled. The ABCD framework [12] is lengthscale independent (e.g. laminate, sub-laminate, laminate). If it is desired, a laminate constructed of 48 plies can be modeled as a single part with 3 elements through the thickness with MAT162. This implementation only works if the material model is calibrated to represent the behavior of a 48 ply sub-laminate. The target application requires calibration of the length scale of interest. The size of the sublaminate depends on the desired tractability of the problem. Ongoing work in NASA ACC Phase II includes the calibration of MAT162 parameters for parts idealized at the sublaminate lengthscale. The plan is to calibrate the material model to represent a [45/90/-45/0] quasi-isotropic sublaminate. This implementation method allows the use of native MAT162 
delamination behavior, foregoing the use of tiebreak contact and improving the tractability of large assembly impact models.

\section{Acknowledgements}

This work was funded by the NASA ACC. The authors wish to acknowledge Brian Kasperson of BR\&T Fast Lean Test Cell and Dr. Mark Pankow of NCSU for their experimental expertise offered to the program.

The material in this paper is based upon work supported by NASA under Award No. NNL09AA00A. Any opinions, findings, and conclusions or recommendations expressed in this material are those of the author(s) and do not necessarily reflect the views of the National Aeronautics and Space Administration.

\section{References}

[1] A. P. K. Joseph, A. M. Waas, W. Ji, E. J. Pineda, S. L. Liguore and S. P. Wanthal, "Progressive Damage and Failure Predictions of Open Hole Tension and Open Hole Compression Specimens," in Proceedings of the 56th AIAA/ASCE/AHS/ASC Structures, Structural Dynamics, and Materials Conference, 2015.

[2] S. P. Engelstad and S. B. Clay, "Comparison of composite damage growth tools for static behavior of notched composite laminates," Journal of Composite Materials, vol. 51, no. 10, pp. 1493-1524, 2016.

[3] S. I. Thorsson, A. Yoshimura, A. M. Waas and M. Rassaian, "Prediction of Low-Velocity Face-On Impact Response of Composite Laminates using High-Fidelity Finite Element Modeling Techniques," in 57th AIAA/ASCE/AHS/ASC Structures, Structural Dynamics, and Materials Conference, San Diego, CA, 2016.

[4] W. Ji, S. P. Sringeri, S. I. Thorsson, C. J. Kosztowny, A. M. Waas and M. Rassaian, "Face-on and Edge-on Impact Response of Composite Laminates," in 56th AIAA/ASCE/AHS/ASC Structures, Structural Dynamics, and Materials Conference, Kissimmee, FL, 2015.

[5] K. J. Hunziker, J. K. Pang, M. E. Melis, M. Pereira and M. Rassaian, "NASA ACC High Energy Dynamic Impact Methodology and Outcomes," in AIAA SCITECH, Kissimmee, Florida, 2018.

[6] A. Byar, J. Iqbal, J. Pang and M. Rassaian, "Determination of Ballistic Limit for IM7/8552 Using MAT261," in AIAA SCITECH, Kissimmee, Florida, 2018.

[7] O. Weckner, F. Cuenca, S. Silling, M. Rassaian and J. Pang, "Determination of Ballistic Limit for IM7/8552 Using Peridynamics," in AIAA SCITECH, Kissimmee, Florida, 2018.

[8] C.-F. Yen, "Ballistic impact modeling of composite materials," in Proceedings of the 7th international LS-DYNA users conference, 2002.

[9] B. Z. Gama, A Short Course on Progressive Composite Damage Modeling in LS-DYNA Using MAT162, Newark, DE: University of Delaware Center for Composite Materials, 2015.

[10] J. Xiao, B. Gama and J. Gillespie, "Progressive Damage and Delamination in Plain Weave S-2 Glass/SC-15 Composites Under Quasi-Static Punch-Shear Loading," Composite Structures, 2007.

[11] B. A. Gama, S. M. Waliul Islam, M. Rahman, J. W. Gillespie Jr., T. A. Bogetti, B. A. Cheeseman, C.-F. Yen and C. P. R. Hoppel, "Punch Shear Behavior of Thick-Section Composites Under Quasi-Static, Low Velocity, and Ballistic Impact Loading," in 50th International SAMPE Symposium \& Exhibition, Long Beach, CA, 2005.

[12] H. Razi, S. Schaefer, S. Wanthal, J. Handler, G. Renieri and B. Justusson, "Rapid Integration of New Analysis Methods in Production," in Thrity-First Technical Conference for The American Society for Composites, Williamsburg, VA, 2015.

[13] M. Pankow, "Through-the-Thickness Reponse of Hybrid 2D and 3D Woven Composites," in 54th AIAA/ASME/ASCE/AHS/ASC Structures, Structural Dynamics, and Materials Conference, 2013.

[14] C.-F. Yen, "A ballistic material model for continuous-fiber reinforced composites," International Journal of Impact Engineering, vol. 46, pp. 11-22, 2012.

American Institute of Aeronautics and Astronautics 


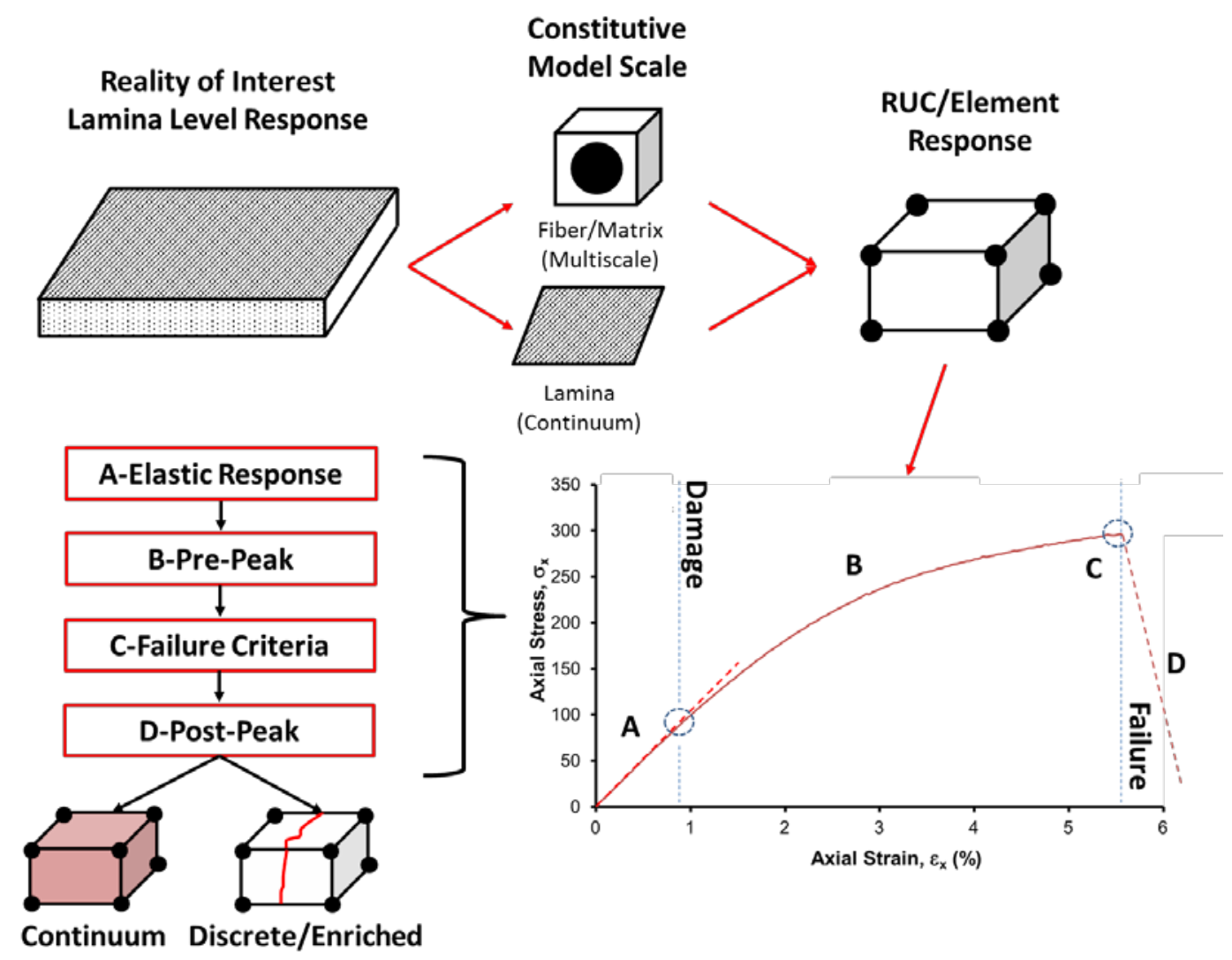

Figure 1: Definition of material models for progressive damage analysis

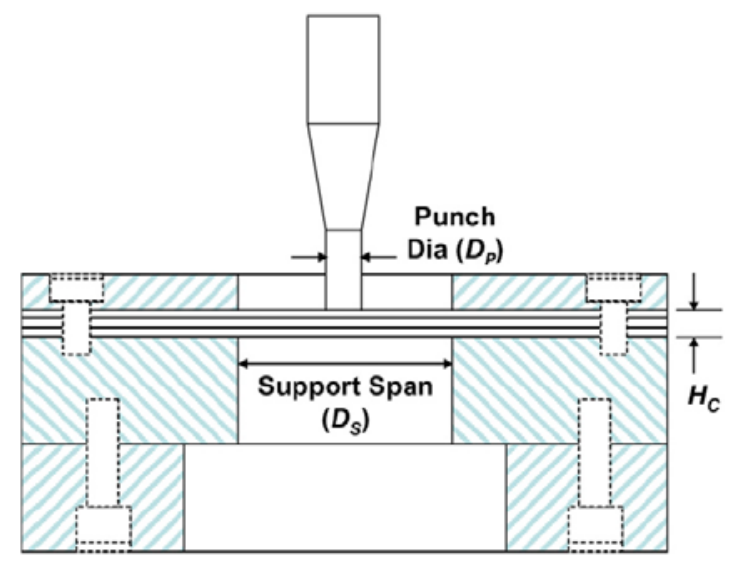

Figure 2: Schematic of the quasi-static punch shear test as proposed by Gama [9] 


\begin{tabular}{|c|c|c|c|c|c|}
\hline Layup & Specimen & $\mathbf{D}_{\mathbf{p}}(\mathbf{i n})$ & $\mathbf{D}_{\mathbf{s}}(\mathbf{i n})$ & SPR & Load Level \\
\hline \multirow{3}{*}[0/90]{$_{4 \mathrm{~s}}$} & 1 & 0.25 & 0 & 0 & Ultimate \\
\cline { 2 - 6 } & 2 & 0.25 & 0 & 0 & Ultimate \\
\cline { 2 - 6 } & 3 & 0.25 & 0 & 0 & Ultimate \\
\cline { 2 - 6 } & 4 & 0.25 & 0 & 0 & Ultimate \\
\cline { 2 - 6 } & 5 & 0.25 & 0 & 0 & Ultimate \\
\hline \multirow{3}{*}[0/90]{$_{4 \mathrm{~s}}$} & 1 & 2.2 & 2.0 & 1.1 & Ultimate \\
\cline { 2 - 6 } & 2 & 2.2 & 2.0 & 1.1 & Ultimate \\
\cline { 2 - 6 }$($ For SFS) & 3 & 2.2 & 2.0 & 1.1 & Ultimate \\
\cline { 2 - 6 } & 4 & 2.2 & 2.0 & 1.1 & Ultimate \\
\cline { 2 - 6 } & 5 & 2.2 & 2.0 & 1.1 & Ultimate \\
\hline
\end{tabular}

Figure 3: Test matrix for quasi-static punch shear

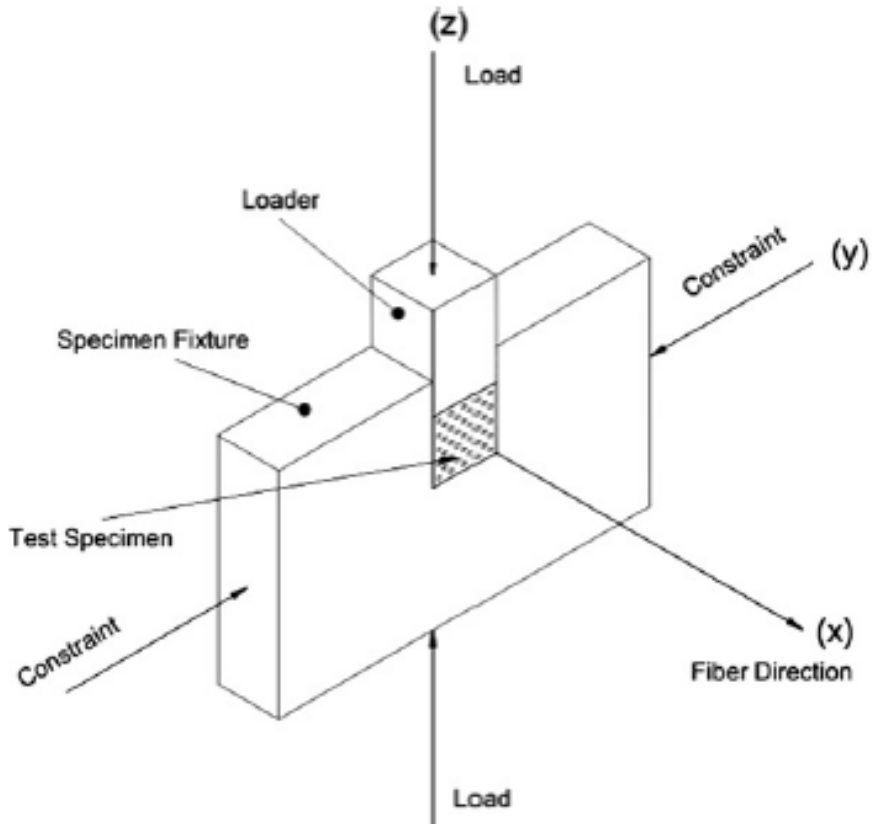

Figure 4: Laterally constrained compression test fixture and unidirectional specimen [13]

Table 1: Comparison of Results for IM7/8552 Failure Parameters Using Two Test Methods

\begin{tabular}{|c|c|c|}
\hline Failure Parameter & QS-PST & LCCT \\
\hline SFS (ksi) & 38.5 & 52.7 \\
\hline SFC (ksi) & 160.6 & 207.6 \\
\hline
\end{tabular}




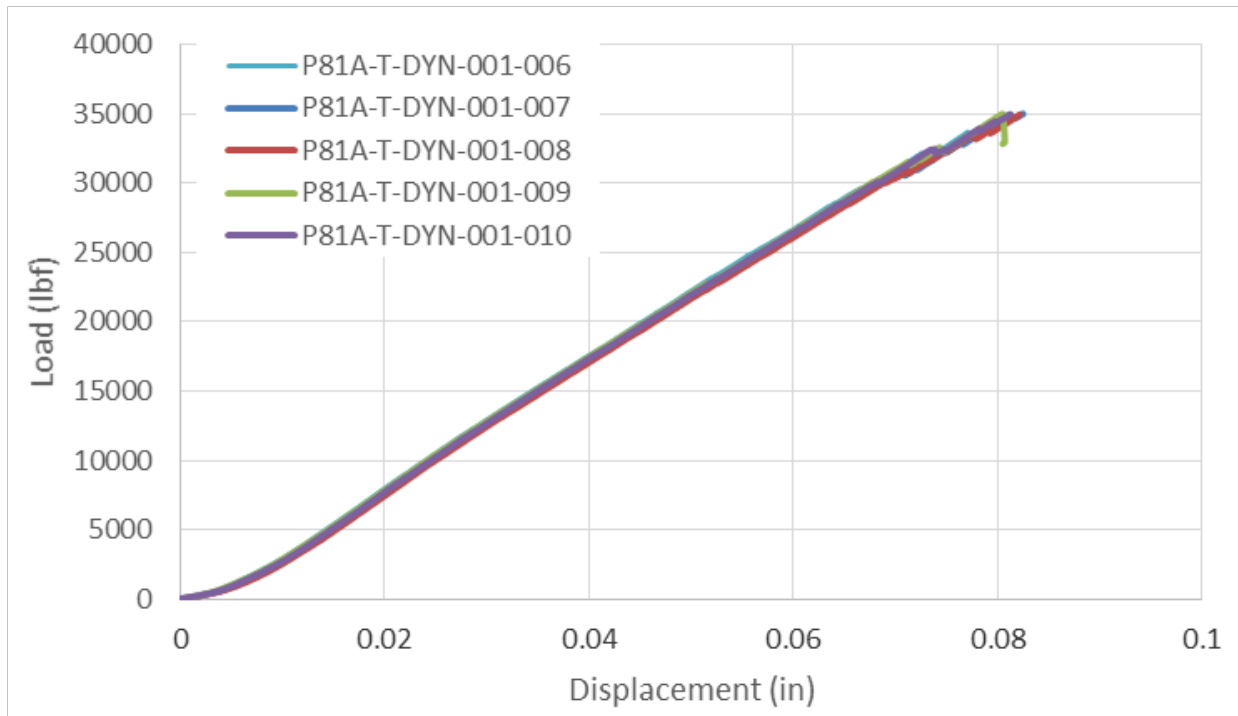

Figure 5: Load-displacement curves for the quasi-static punch shear with $\mathrm{SPR}=\mathbf{0 . 0}$

Table 2: Tabulated QS-PST Data (SPR $=0.0$ )

\begin{tabular}{|c|c|c|c|}
\hline Specimen & Press Area & Failure Load & SFC \\
\hline ID & {$\left[\mathrm{in}^{2}\right]$} & [lbf] & [ksi] \\
\hline 6 & \multirow{5}{*}{0.19635} & 32206 & 164.0 \\
\hline 7 & & 30872 & 157.2 \\
\hline 8 & & 30874 & 157.2 \\
\hline 9 & & 31532 & 160.6 \\
\hline 10 & & 32433 & 165.2 \\
\hline \multicolumn{2}{|c|}{ average } & 31583 & 160.9 \\
\hline \multicolumn{2}{|c|}{ stdev } & 728 & 3.7 \\
\hline
\end{tabular}

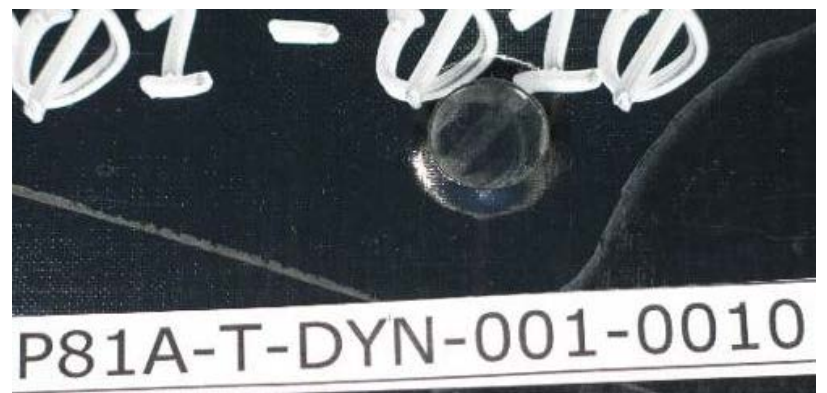

Figure 6: Tested sample for the quasi-static punch shear with SPR=0.0 


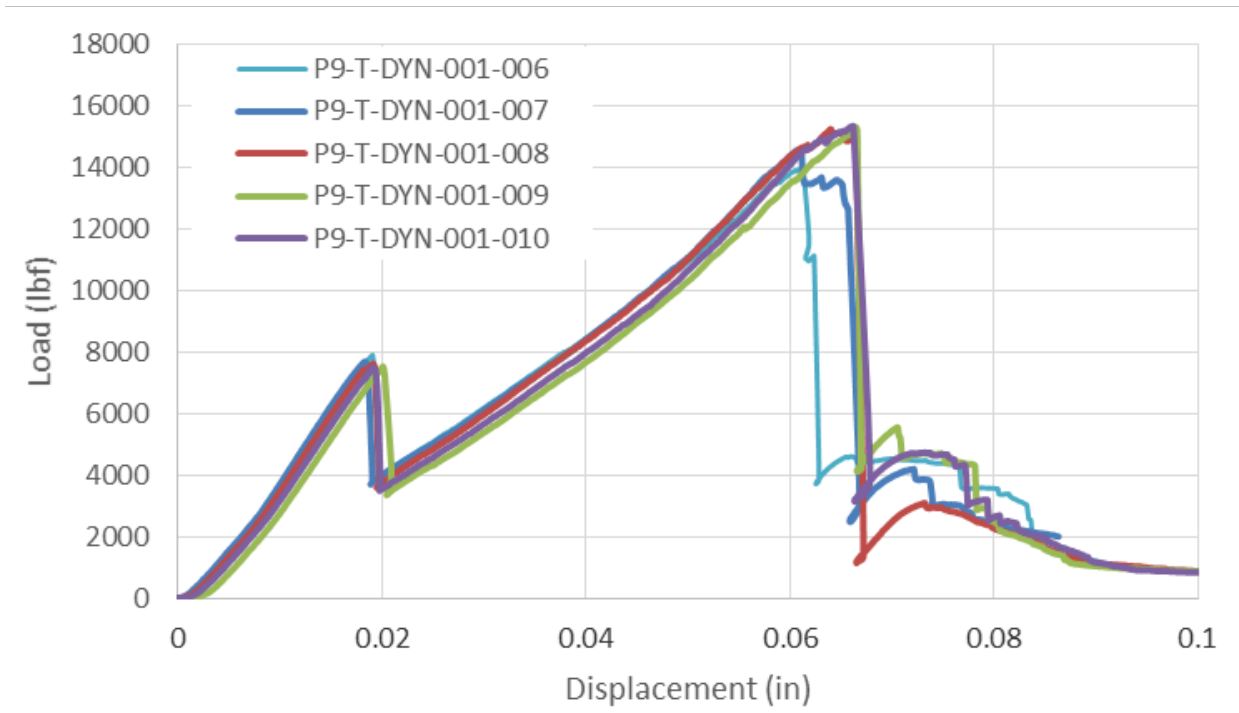

Figure 7: Load-displacement curves for the quasi-static punch shear with SPR=1.1

Table 3: Thicknesses of Lip Developed as a Result of Delamination

\begin{tabular}{|c|c|c|c|c|c|c|}
\hline & & \multicolumn{3}{|c|}{ LIP THRU THICKNESS (IN) } & \multirow{2}{*}{ AVG } \\
\cline { 3 - 5 } COUPON & SPR & $\mathbf{1}$ & $\mathbf{2}$ & $\mathbf{3}$ & AVG \\
\hline P9-T-DYN-001-006 & 1.1 & 0.0505 & 0.0460 & 0.0510 & 0.0492 \\
\hline P9-T-DYN-001-007 & 1.1 & \multicolumn{3}{|c|}{ did not punch out } & -- \\
\hline P9-T-DYN-001-008 & 1.1 & 0.0540 & 0.0430 & 0.0480 & 0.0483 \\
\hline P9-T-DYN-001-009 & 1.1 & 0.0525 & 0.0595 & 0.0485 & 0.0535 \\
\hline P9-T-DYN-001-0010 & 1.1 & \multicolumn{3}{|c|}{ did not punch out } & -- \\
\hline
\end{tabular}

Table 4: Tabulated QS-PST Data (SPR=1.1)

\begin{tabular}{|c|c|c|c|c|c|}
\hline Specimen & Press Circum. & Plug Thickness & Plug Area & Failure Load & SFS \\
\hline ID & [in] & [in] & {$\left[\mathrm{in}^{2}\right]$} & [lbf] & [ksi] \\
\hline 6 & \multirow{5}{*}{5.7177} & 0.06875 & 0.393092 & 13993 & 35.6 \\
\hline 7 & & \multicolumn{2}{|c|}{ Did not punch out } & 14567 & -- \\
\hline 8 & & 0.06965 & 0.398238 & 15221 & 38.2 \\
\hline 9 & & 0.06445 & 0.368506 & 15313 & 41.6 \\
\hline 10 & & \multicolumn{2}{|c|}{ Did not punch out } & 15345 & -- \\
\hline \multicolumn{2}{|c|}{ average } & 0.06762 & 0.38661 & 14888 & 38.5 \\
\hline \multicolumn{2}{|c|}{ stdev } & 0.00278 & 0.01589 & 593 & 3.0 \\
\hline
\end{tabular}




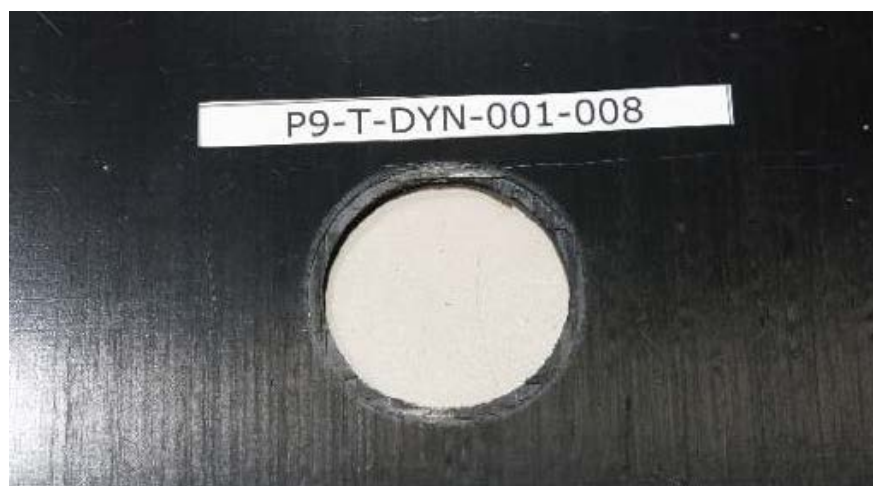

Figure 8: Tested sample for the quasi-static punch shear with $S P R=1.1$

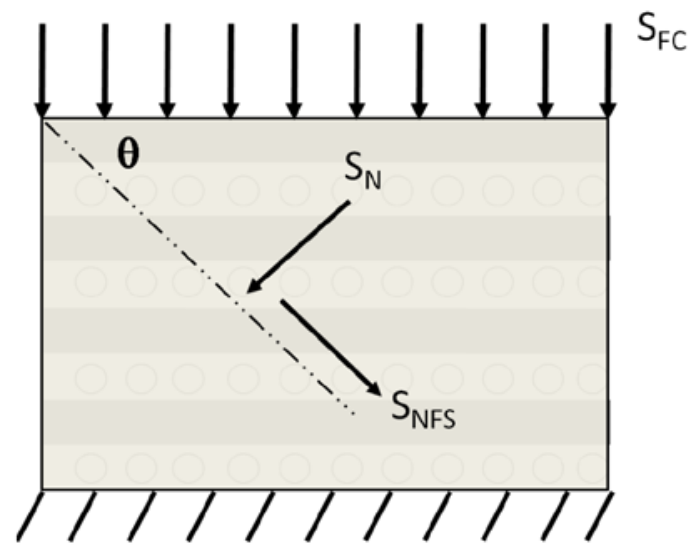

Figure 9: Schematic of failed LCCT coupon proposed by Pankow [13]

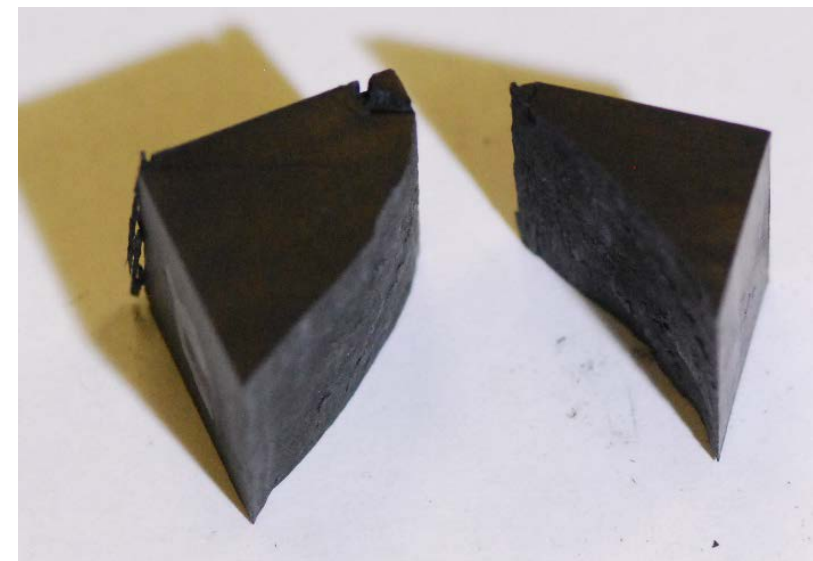

Figure 10: Tested LCCT sample exhibiting characteristic shear plane failure 


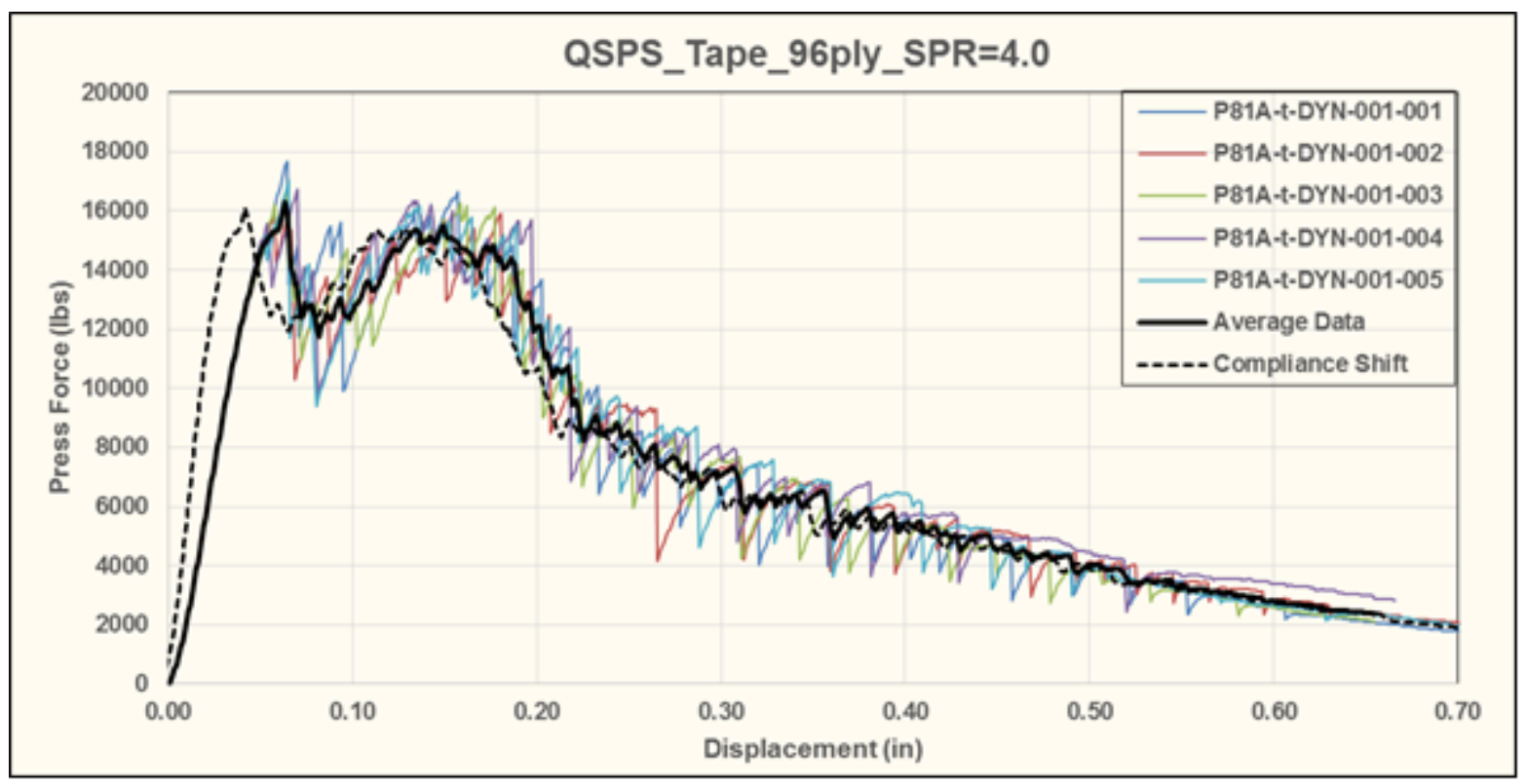

Figure 11: Quasi-static punch shear test results

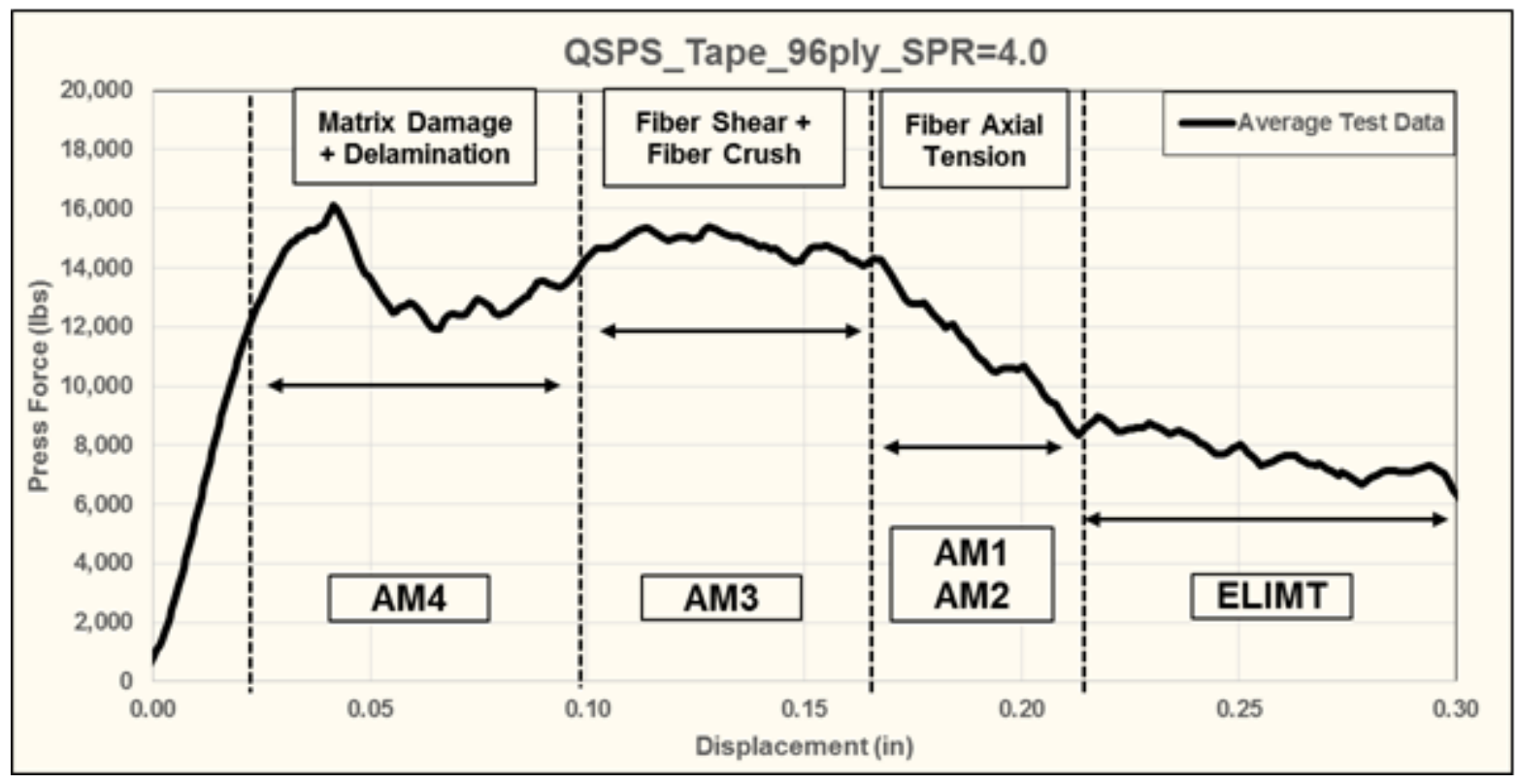

Figure 12: Sequential progression of damage modes and associated MAT162 calibration parameters 


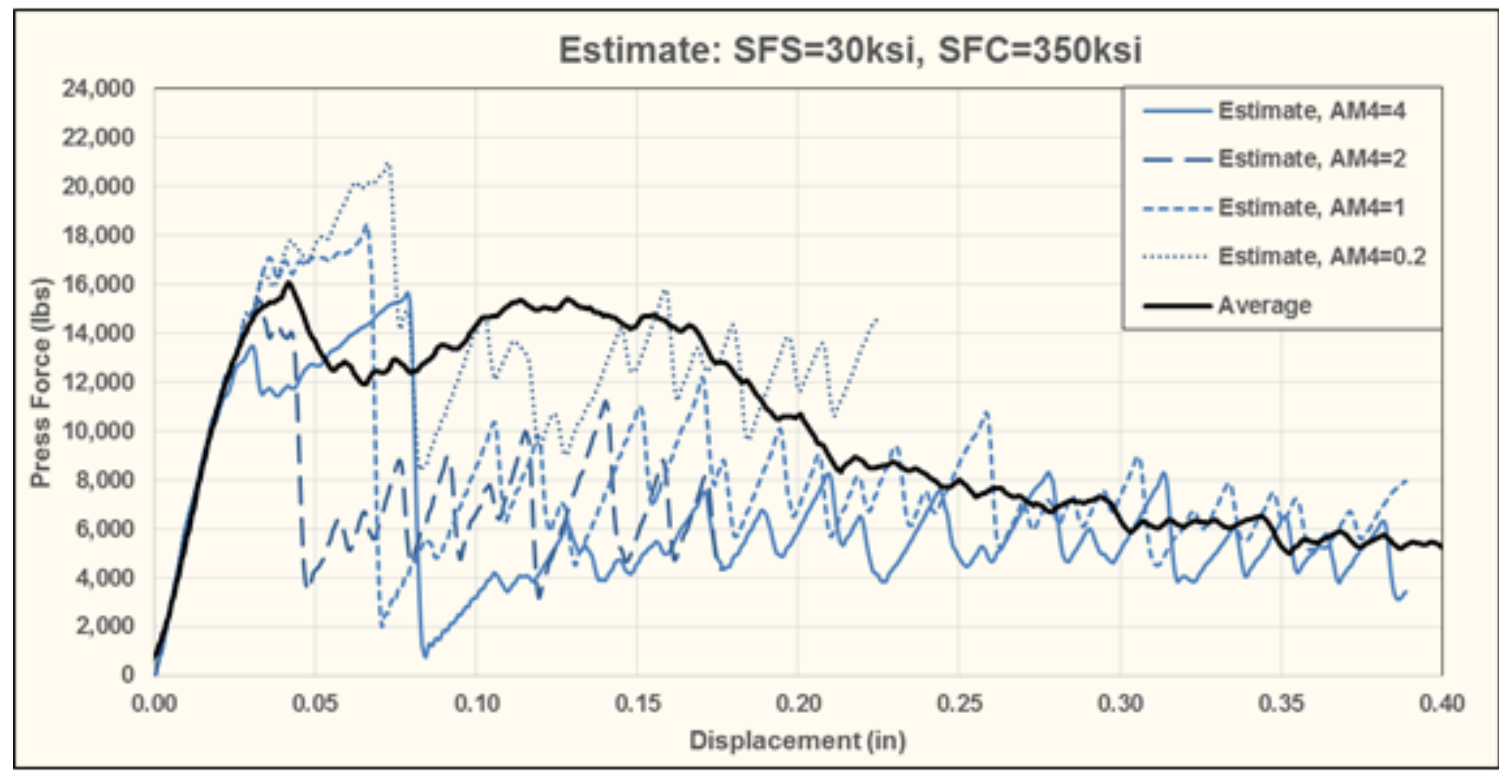

Figure 13: QSPS model results of AM4 softening parameter calibration using estimated values for SFS and SFC stress limits (Compliance adjusted average test results for reference)

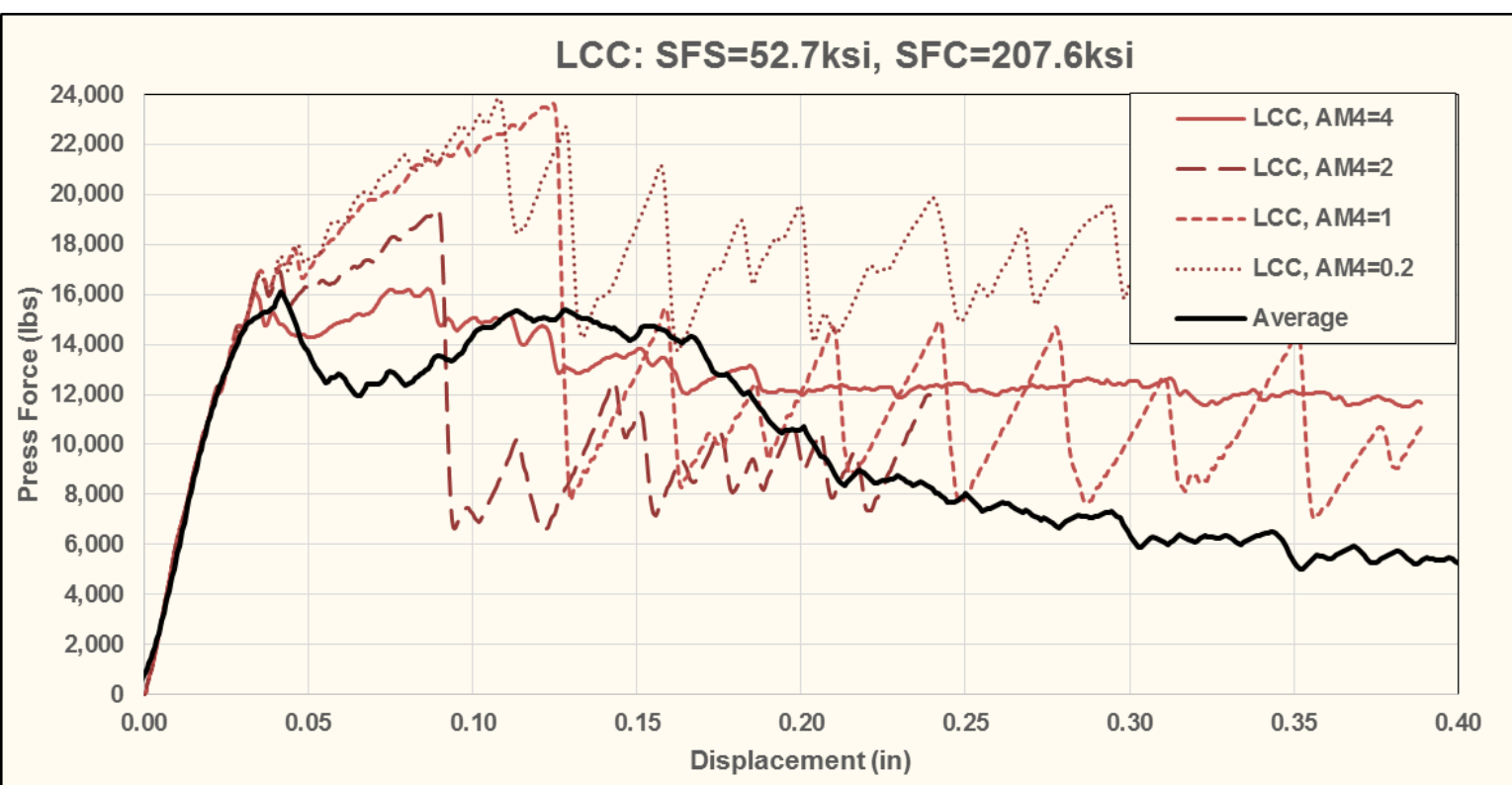

Figure 14: QSPS model results of AM4 softening parameter calibration using values for SFS and SFC stress limits obtained from LCC testing (Compliance adjusted average test results for reference) 


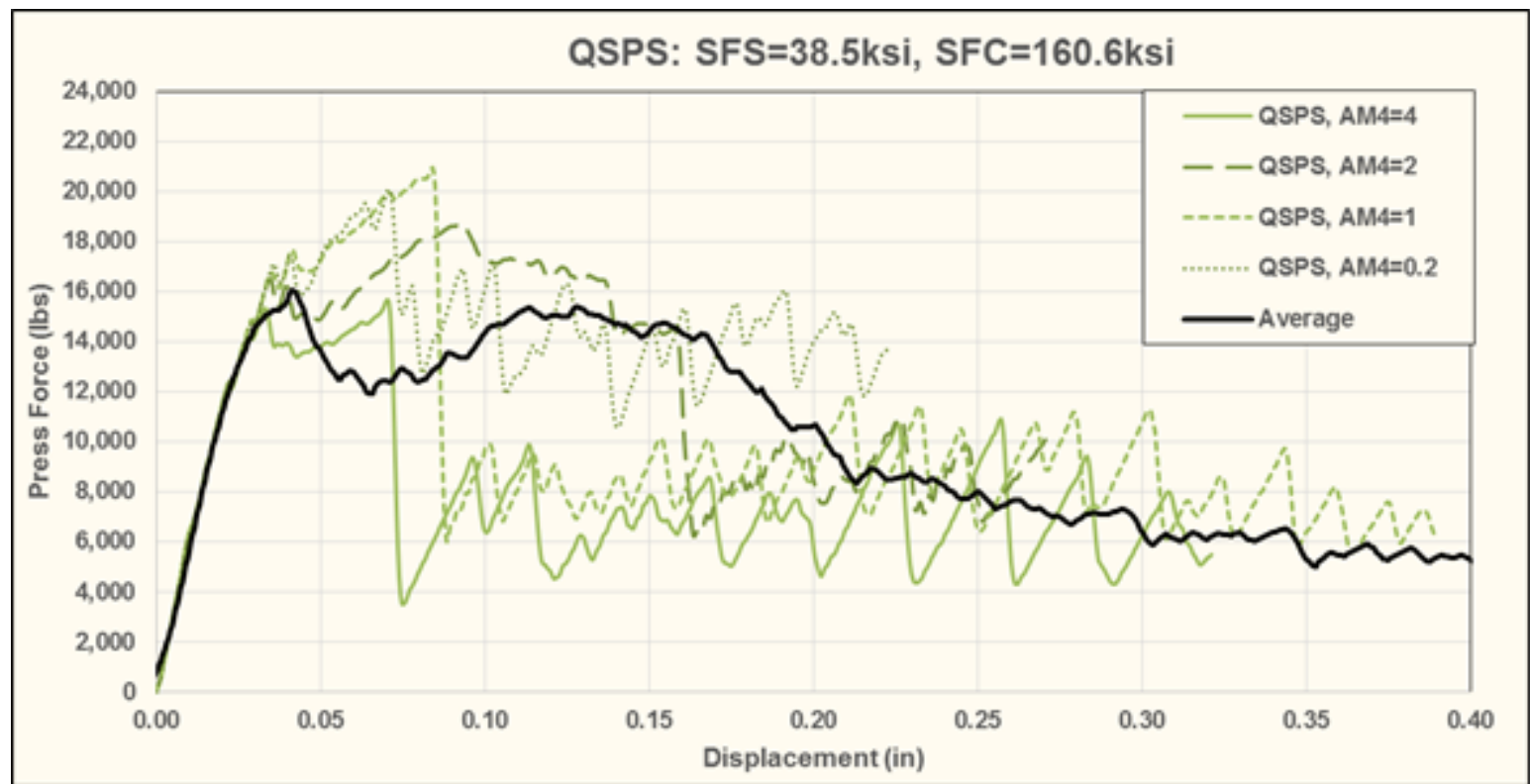

Figure 15: QSPS model results of AM4 softening parameter calibration using values for SFS and SFC stress limits obtained from modified QSPS testing (Compliance adjusted average test results for reference)

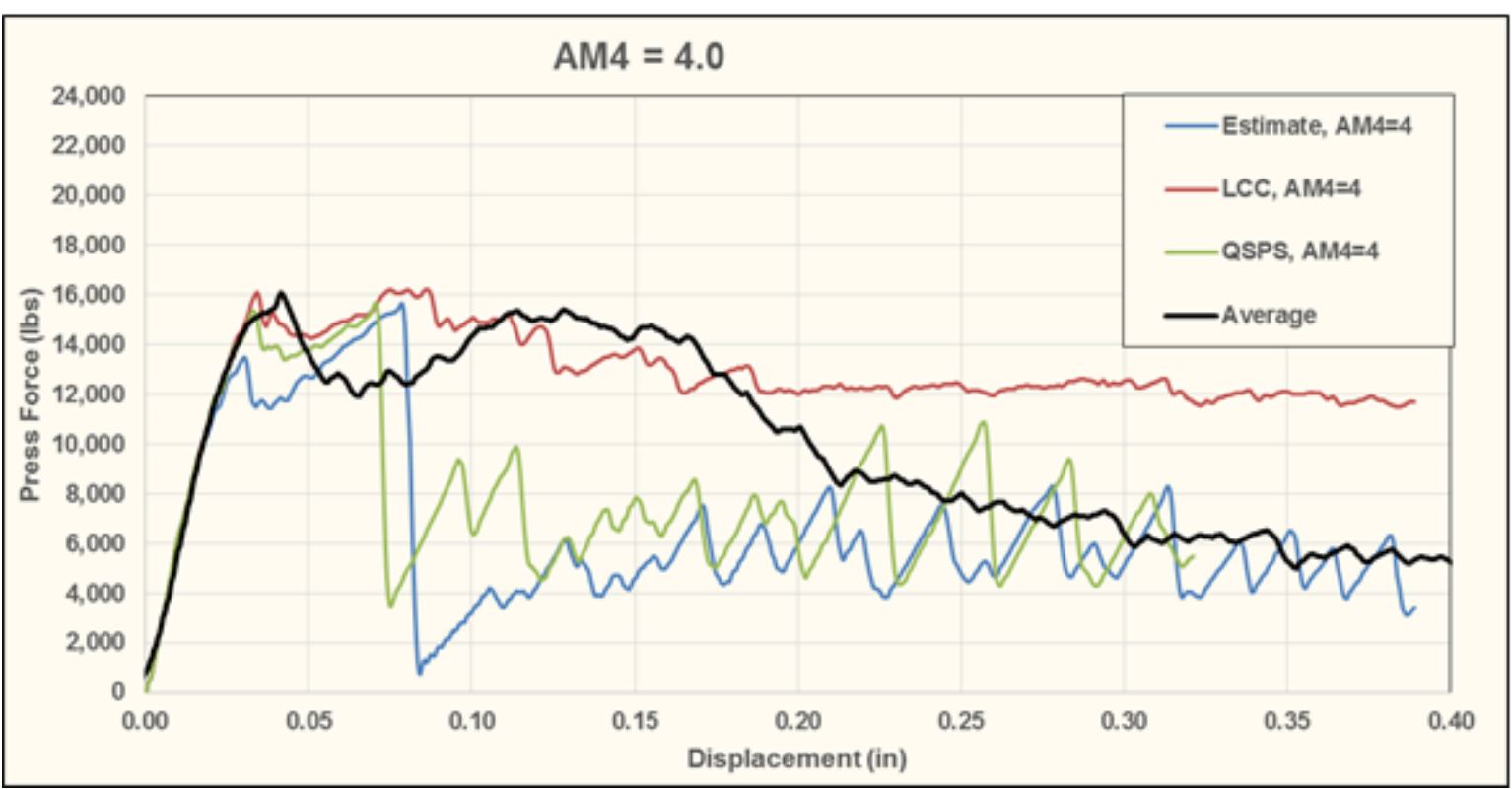

Figure 16: QSPS model results comparing effect of SFS and SFC stress limits obtained from multiple methods (Compliance adjusted average test results for reference) 


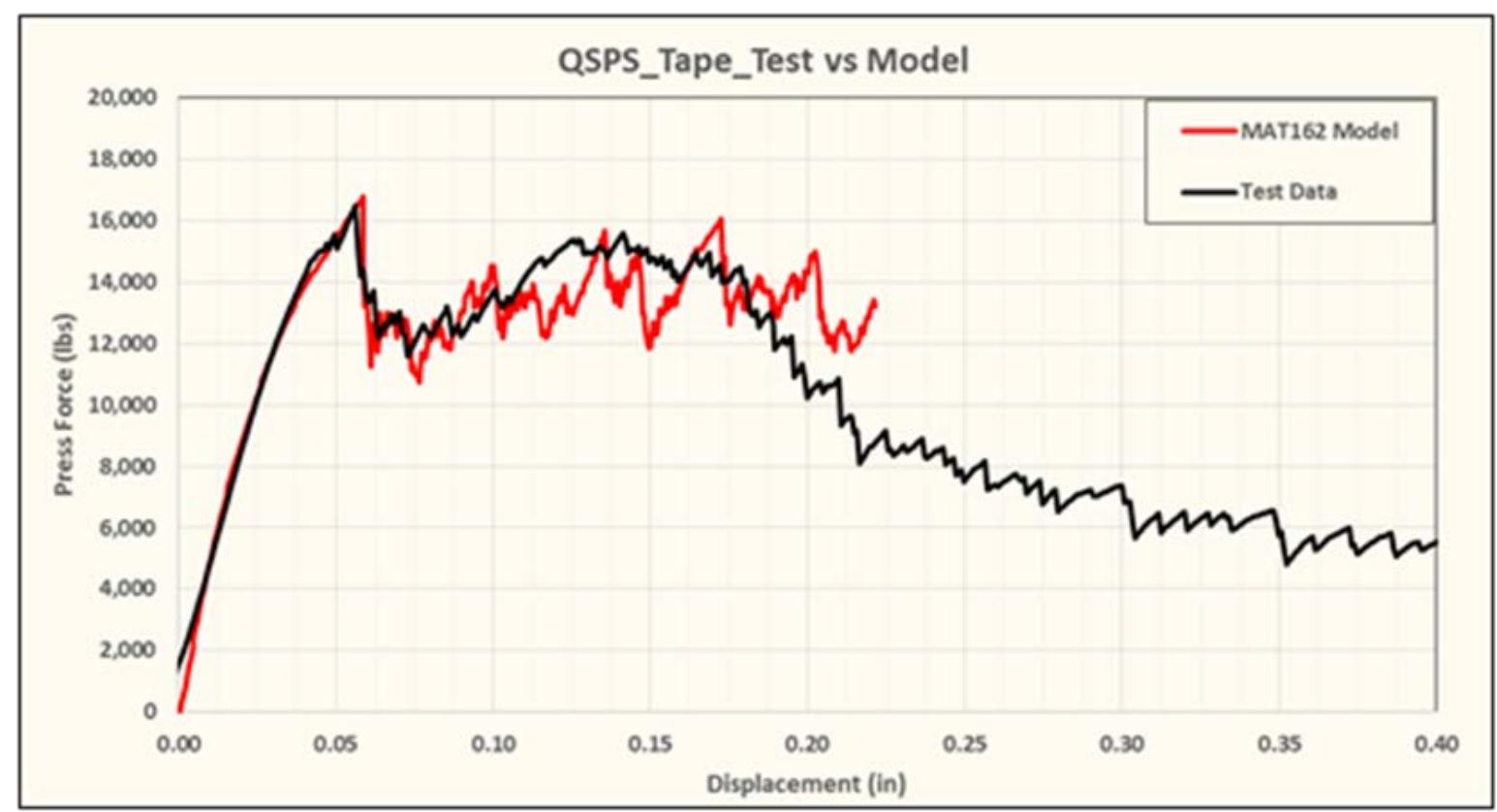

Figure 17: Calibrated MAT162 model results vs. QS-PST data 